Background Inattention to symptoms of mental disorders and substance abuse in patients with HIV/AIDS and other at risk groups, may lead to irreversible damages. Present research aimed at comparison of psychopathological dimensions in substance abusers with and without HIV/AIDS and healthy matched groups.

Methods In a cross-sectional and analytical study, selected samples by available, consecutive, and objective methods were $43 \mathrm{HIV}$ positive substance abusers, 49 HIV negative substance abusers under Methadone Maintenance Therapy (MMT) in counselling clinic of behavioural diseases and addiction abandonment and 45 ordinary individuals. All of them were evaluated by matched confounding variables via symptom check list-90-Revised (SCL-90-R). Results analysed by chi-square $\left(\chi^{2}\right)$, independent $t$ test, one-way analysis of variance (ANOVA) and Gabriel post hoc.

Results Findings indicated significant difference between these groups in Global Severity Index (GSI), Positive Symptom Distress Index (PSDI) and Positive Symptom Total (PST) (P < 0.0001). Two by two comparison of the three groups from psychopathological dimensions revealed that substance abusers with HIV/AIDS persistently suffer more mental problems in all dimensions compared with healthy ones $(\mathrm{P}<0.05)$ and in other dimensions including somatization, interpersonal sensitivity, depression, anxiety, phobia, and psychoticism in comparison with HIV negative substance abusers $(\mathrm{P}<0.05)$. Yet, the difference in paranoid ideation, hostility and obsessive-compulsive case was insignificant. Two by two comparison of healthy ones and substance abusers without HIV/AIDS showed higher levels of depression and Psychoticism in substance abusers $(\mathrm{P}<0.05)$ but no difference in other dimensions.

Conclusion Comorbidity of substance abuse and HIV diagnosis intensify mental disorders symptoms. Moreover, lack of prevention and implementation of appropriate psychological and psychiatric interventions after substance abuse and HIV lead to extended establishment of mental disorders symptoms. Some implications of these results are discussed results may direct future researches.

\section{P3.314 CAN CHLAMYDIA PREVALENCE MONITORING DATA BE USED TO EVALUATE IMPACT OF SCREENING? THE US CDC INFERTILITY PREVENTION PROJECT EXPERIENCE}

doi:10.1136/sextrans-2013-051184.0768

'K T Bernstein, 'S Stephens, ${ }^{2}$ E Torrone, ${ }^{3} \mathrm{~J}$ Chow, 'S Philip. 'San Francisco Department of Public Health, San Francisco, CA, United States; ${ }^{2}$ Centers for Disease Control, Atlanta, CA, United States; ${ }^{3}$ UCSF, San Francisco, CA, United States

Background Chlamydia testing data are often used for prevalence monitoring to evaluate screening programmes; however, trends in positivity are impacted by changes in screening coverage and criteria, independent of changes in population prevalence, and thus are difficult to interpret. Given limited resources, many chlamydia control programmes in the US target screening to improve cost-effectiveness. We explored the potential impact of focusing screening on high prevalence (i.e., > 3\%) clinics on trends in chlamydia positivity. Methods We analysed line-listed data on women tested for chlamydia in family planning clinics participating in the Infertility Prevention Project (IPP) during 2000-2011. Trends in annual positivity in family planning clinics participating in IPP who reported at least 120 tests restricted to patients aged 15-24 years were examined among two cohorts: (1) all clinics and (2) all clinics, with testing data removed from subsequent years for clinics where positivity fell below 3\%.

Results Positivity trends for both cohorts are shown in the table, along with overall percent change in positivity over the period. All trend lines increased over time; however, trend lines with low prevalence clinics removed had a higher positivity at each year. Similar patterns were seen for both cohorts of clinics, as well as when stratified by geographic region. Percent change in positivity over the 12 year period was $52.2 \%$ for cohort 1 and $64.1 \%$ in cohort 2 .

Conclusion Our analysis of chlamydia data suggests that individual point estimates of chlamydia positivity are likely overestimated when chlamydia screening was targeted to high prevalence clinics; however trends over time were similar in the two analytic groups. Data used for programme monitoring and evaluation may bias point estimates of prevalence. Caution should be used when using prevalence monitoring data to evaluate impact of screening without considering clinic-level confounders.

YEAR All Clinics Below 3\% Removed

P3.315 DOES ADJUSTING FOR SEXUAL BEHAVIOUR IMPROVE
THE ACCURACY OF POPULATION-BASED CHLAMYDIA
INCIDENCE AND SCREENING RATES AMONG
ADOLESCENTS IN BRITISH COLUMBIA, CANADA?

doi:10.1136/sextrans-2013-051184.0769

1.2 M Gilbert, ${ }^{3} \mathrm{~A}$ Roberts, ${ }^{3} \mathrm{~K}$ Mitchell, ${ }^{4} \mathrm{Y}$ Homma, ${ }^{5} \mathrm{C}$ Warf, ${ }^{4} \mathrm{~L}$ Daly, ${ }^{4,5}$ E Saewyc. ${ }^{1}$ British Columbia Centre for Disease Control, Vancouver, BC, Canada; ${ }^{2}$ School of Population and Public Health, University of British Columbia, Vancouver, BC, Canada; ${ }^{3}$ Department of Pediatrics, $B C$ Children's Hospital/University of British Columbia, Vancouver, $B C$, Canada; ${ }^{4}$ School of Nursing, University of British Columbia, Vancouver, BC, Canada; ${ }^{5}$ Division of Adolescent Health and Medicine, BC Children's Hospital/University of British Columbia, Vancouver, BC, Canada

Background Recent studies using setting-specific health insurance or clinical datasets have demonstrated the importance of considering

Abstract P3.314 Table 1

\begin{tabular}{|c|c|c|c|c|c|c|c|c|}
\hline \multirow[b]{2}{*}{ YEAR } & \multicolumn{4}{|l|}{ All Clinics } & \multicolumn{4}{|c|}{ Below 3\% Removed } \\
\hline & Point Estimate & $95 \% \mathrm{CI}$ & & Testing Volume & Point Estimate & $95 \% \mathrm{CI}$ & & Testing Volume \\
\hline 2000 (baseline) & $5.77 \%$ & $5.72 \%$ & $5.82 \%$ & 852274 & $5.77 \%$ & $5.72 \%$ & $5.82 \%$ & \\
\hline 2001 & $5.79 \%$ & $5.74 \%$ & $5.83 \%$ & 904906 & $6.32 \%$ & $6.26 \%$ & $6.37 \%$ & 750,924 \\
\hline 2002 & $6.02 \%$ & $5.98 \%$ & $6.07 \%$ & 923056 & $6.61 \%$ & $6.55 \%$ & $6.66 \%$ & 752,666 \\
\hline 2003 & $6.37 \%$ & $6.32 \%$ & $6.42 \%$ & 949916 & $7.09 \%$ & $7.03 \%$ & $7.15 \%$ & 750,525 \\
\hline 2004 & $6.99 \%$ & $6.94 \%$ & $7.04 \%$ & 976398 & $7.80 \%$ & $7.74 \%$ & $7.86 \%$ & 756,625 \\
\hline 2005 & $6.91 \%$ & $6.86 \%$ & $6.96 \%$ & 985064 & $7.68 \%$ & $7.62 \%$ & $7.74 \%$ & 768,165 \\
\hline 2006 & $7.11 \%$ & $7.06 \%$ & $7.17 \%$ & 990214 & $7.99 \%$ & $7.93 \%$ & $8.05 \%$ & 762,244 \\
\hline 2007 & $7.61 \%$ & $7.55 \%$ & $7.66 \%$ & 912142 & $8.53 \%$ & $8.47 \%$ & $8.60 \%$ & 701,221 \\
\hline 2008 & $7.97 \%$ & $7.92 \%$ & $8.03 \%$ & 887940 & $8.95 \%$ & $8.88 \%$ & $9.02 \%$ & 685,459 \\
\hline 2009 & $8.18 \%$ & $8.13 \%$ & $8.24 \%$ & 897558 & $9.16 \%$ & $9.09 \%$ & $9.23 \%$ & 693,921 \\
\hline 2010 & $8.34 \%$ & $8.29 \%$ & $8.40 \%$ & 950214 & $9.09 \%$ & $9.03 \%$ & $9.16 \%$ & 749,957 \\
\hline 2011 & $8.78 \%$ & $8.72 \%$ & $8.84 \%$ & 896104 & $9.47 \%$ & $9.40 \%$ & $9.54 \%$ & 710,947 \\
\hline Percent change from 2000-2011 & $52.2 \%$ & $52.4 \%$ & $51.9 \%$ & & $64.1 \%$ & $64.3 \%$ & $63.9 \%$ & \\
\hline
\end{tabular}


sexual activity when calculating Chlamydia incidence and screening rates, particularly for adolescents. Using data from a provincial adolescent health survey we assessed the impact of adjusting for sexual activity on population-based Chlamydia incidence and screening rates among adolescents in British Columbia (BC), Canada.

Methods We estimated the proportion of adolescent males (15-18 years) and females (14-18 years) who had ever had sexual intercourse (i.e., sexually active) using data from a cluster-stratified survey of public school students (Grades 7-12) completed by 30,000 BC students in 2003 and 2008. Using provincial Chlamydia surveillance and testing data we compared adolescent Chlamydia screening and incidence rates in BC by age and gender, using total and sexually active populations as denominators.

Results During these time periods, an estimated $32 \%$ and $33 \%$ of males $15-18$ years and $28 \%$ and $31 \%$ of females $14-18$ years were sexually active in 2003 and 2008 respectively. Regardless of denominator used, screening and incidence rates increased with age, and were higher among females compared to males. Sexually active incidence and screening rates were consistently higher with a more pronounced impact at younger ages. For example, in 2008 screening rates among 14 year old females were $26.2 \%$ vs $2.5 \%$ in sexually active and total populations respectively, while the corresponding rates among 18 year old females were $60.2 \%$ vs $28.9 \%$ (2.1 times higher).

Conclusions Using data representing the entire population of $\mathrm{BC}$ adolescents we demonstrated that without adjustment for sexual behaviour, adolescent Chlamydia incidence and screening rates are substantially under-estimated, particularly at younger ages. Adjusting for sexual behaviour using population survey data is essential for accurately monitoring the population impact of prevention and screening programmes among adolescents.

\section{P3.316 ESTIMATING CHLAMYDIA AND GONORRHOEA BURDEN WITHIN THE US ARMY - A REVIEW OF PASSIVE SURVEILLANCE SYSTEMS TO IDENTIFY INCIDENT INFECTIONS}

doi:10.1136/sextrans-2013-051184.0770

'N N Jordan, ${ }^{1,2} \mathrm{~N} \mathrm{~K}$ Leamer, ${ }^{3} \mathrm{G}$ Nowak, ${ }^{4} \mathbf{J}$ C Gaydos. ${ }^{1}$ US Army Institute of Public Health, Aberdeen Proving Grounds-South, MD, United States; ${ }^{2}$ Oak Ridge Institure of Science and Education, Oak Ridge, TN, United States; 'Navy Marine Corps Public Health Center, Norfolk, VA, United States; ${ }^{4}$ Armed Forces Health Surveillance Center, Silver Spring, MD, United States

Background The burden of sexually transmitted infections (STI) within the US military is primarily assessed through review of case reports for notifiable conditions (e.g., Chlamydia, gonorrhoea, and syphilis). The degree to which under-reporting affects burden approximations is unknown. This study was conducted to assess the burden of Chlamydia and gonorrhoea infections and compare case capture across multiple military medical data systems.

Methods Incident infections among Army active duty soldiers from January 2007-December 2011 were identified using three data sources: Health Level 7 laboratory records (HL7), Military Health System Data Repository (MDR) medical records, and case reports from the Disease Reporting System internet (DRSi) and its predecessor, the Reportable Medical Event System (RMES). A thirty day rule was used to define incident cases; i.e., successive records logged within 30 days were excluded.

Results 53,228 incident Chlamydia infections and 11,065 incident gonorrhoea infections were identified over five years; calendar year 2011 incidence rates were 22.0 and 4.2 infections per 1000 personyears, respectively. Chlamydia case capture was $80 \%, 74 \%$, and $35 \%$, respectively for case reports, HL7, and medical records. Gonorrhea case capture was $65 \%, 62 \%$, and $56 \%$, respectively for medical records, case reports and HL7. A notable decrease in laboratory identified infections occurred in 2011, decreasing from highs of $78 \%$ and
$60 \%$ in 2008 to $67 \%$ and $49 \%$ for Chlamydia and gonorrhoea, respectively.

Conclusion The results emphasise the need to utilise multiple databases to obtain more complete estimates of STI burden among active duty Army personnel. While this comprehensive approach provides a better approximation of burden, estimates are likely conservative since many infections go undetected or undocumented. Future analyses should incorporate advanced statistical methods such as capture-recapture methodology to estimate infections not identified through conventional passive surveillance. Furthermore, the decreased case detection through laboratory records is notable, and worthy of investigation.

\section{P3.317 USE OF TABLETS FOR DATA COLLECTION AMONG FEMALE SEX WORKERS: LESSONS LEARNED FROM A BEHAVIOURAL SURVEILLANCE STUDY IN HONDURAS, 2012}

doi:10.1136/sextrans-2013-051184.0771

${ }^{1} \mathbf{N}$ Farach, ${ }^{2 \mathrm{H}}$ Galindo, ${ }^{3} \mathrm{~F}$ Tinajeros, ${ }^{4} \mathrm{M}$ Guardado. ${ }^{1} \mathrm{CDC} / \mathrm{CAR} / \mathrm{DGHA}$, Tegucigalpa, Honduras, ${ }^{2}$ Secretaria de Salud, Tegucigalpa, Honduras, ${ }^{3}$ TEPHINET, Tegucigalpa, Honduras, 4TEPHINET, Guatemala, Guatemala

Background The use of tablets and other hand-held devices is increasingly considered as an option for survey data collection, particularly sensitive personal information among key populations.

Methods In 2012, a behavioural surveillance study was conducted among 1426 female sex workers (FSWs) in Honduras. Sensitive personal information was collected, including sexual practises, condom use, sexual violence, alcohol and drug use, and reporting of STI symptoms. Research instruments were inputted into tablets and piloted with FSWs. During the study, facilitators briefed participants in tablet use while participants inputted their responses, in case they had any issues. Daily occurrences were recorded into a log. We analysed qualitative pilot results reports and discussed the advantages and disadvantages of using tablets for data collection with survey facilitators and investigators during regular monitoring visits.

Results Tablet use eliminated the need for paper questionnaires and data entry, and allowed for early database population as surveys were uploaded to a virtual platform. Facilitator help was repeatedly required by participants and self-administered surveys often took more time to complete, particularly during the first sections when participants were familiarising themselves with the tablet. Issues with web connectivity prevented data submission as scheduled in some sites and two tablets were reported missing in one site (out of a total of 22 tablets in the study).

Conclusions The use of tablets can reduce certain study costs (data input, photocopying) and facilitate data management. Facilitators should be available to assist survey participants as they input their own responses, particularly in settings where participants have had little exposure to technology. A brief introduction to tablet use and practise session for participants should be incorporated into the survey flow. Adequate measures should be taken to safeguard tablets and a stable internet connexion should be guaranteed.

\section{P3.319 MALIGNANCIES SURVEILLANCE IN A HISPANIC HIV/AIDS COHORT BEFORE AND AFTER HAART}

doi:10.1136/sextrans-2013-051184.0772

'A M Mayor, 'D M Fernandez, 'E Rios-Oliveras, ${ }^{2} \mathrm{D}$ Zavala, 'R F Hunter-Mellado. ${ }^{1}$ Retrovirus Research Center, Universidad Central del Caribe, Bayamon, Puerto Rico, 2Puerto Rico Central Cancer Registry, San Juna, Puerto Rico

Background This study describes trends in malignancies as it relates to the availability of highly active antiretroviral therapy (HAART) in a Hispanic HIV/AIDS infected cohort.

Methods The database of 4,232 HIV infected adults followed in the Retrovirus Research Center at Bayamon, Puerto Rico was 